\title{
NIÑAS MADRES EN CHILE: UN PROBLEMA QUE SIGUE SIN SOLUCIÓN
}

El Profesor Hugo Behm nos mostraba en su clásico libro "Mortalidad Infantil y Nivel de Vida", publicado en 1962, que en el Chile de 1959 la mortalidad infantil era de 84 muertes de menores de un año por 1000 nacidos vivos en aquellos niños que recibían atención médica y que subía a 147 por 1000 nacidos vivos en aquellos que no la recibían, es decir un $75 \%$ más.

Casi 50 años después, en el Chile de 2007 tenemos otra comparación: la mortalidad infantil a nivel nacional fue de 8,5 por 1000 nacidos vivos, pero en los hijos de niñas-madres de 14 años y menos, fue de 18,9 por 1000 nacidos vivos, es decir más del doble, mientras que en madres de 15 a 19 años alcanzaba a 10,7 por 1000 nacidos vivos. Todos estos niños, hijos de madres adolescentes, habían tenido atención médica y por lo tanto la gravedad de las patologías o la repetición de ellas, o el retraso en la consulta, seguramente son los factores condicionantes de estas diferencias. En la mortalidad neonatal hay un perfil muy parecido.

En 50 años el problema ha cambiado drásticamente. Lo que ayer era un problema casi sin solución, solamente reemplazando las muertes infantiles con altas natalidades, hoy está la fecundidad de alto riesgo que se instala en las adolescentes de menor edad, con una estela de daño y muerte que no corresponde al nivel de desarrollo que Chile quiere.

La mortalidad materna en las niñas-madres menores de 15 años se ha mantenido en 0 desde el año 2000 hasta ahora, pero en las adolescentes de 15 a 19 años es comparable a la mortalidad materna a toda edad. La calidad profesional en la atención del parto y el control prenatal han incidido en estos resultados.

La vulnerabilidad de los hijos de niñas-madres se refleja en el riesgo de parto prematuro. Un análisis desde 1995 a 2004 en Chile, aún no publicado, revela que los riesgos son mayores de tener un recién nacido vivo menor de 2.500 gramos y de 36 semanas de gestación, a medida que desciende la edad de la madre. Este indicador, en las madres de 20 a 24 años presentó una frecuencia de $2,9 \%$, alcanzando a $8,6 \%$ en las madres de 11 y 12 años, con OR de 3,1 y en las de 13 años de $2,4 \%$ con OR de 1,8 .

También sabemos que la frecuencia de consultas por accidentes infantiles y episodios de enfermedad, son significativamente más altos en los hijos de niñas-madres, y que en Chile los factores de riesgo predictivo de tener un aborto en la adolescencia se asocia a la primera relación sexual entre los 12 y 14 años (OR 5,8) o el primer embarazo entre los 13 y 15 años (OR 4,3).

Todas las evidencias revelan que la vulnerabilidad del embrión, feto o recién nacido, es mayor en los embarazos ocurridos en las niñas-madres. Las evidencias nos llevan a decir que un embarazo en una adolescente sigue siendo de riesgo y especialmente si este ocurre en una niña menor de 15 años. El deseo de maternidad en las adolescentes, como sustituto de la ausencia de proyectos de vida o de autoestima, no es un reflejo de un deseo innato, es el sustituto de la ausencia de afectos y comunicación. Es la expresión de querer ser alguien o sentirse alguien, en un medio deprimido por la miseria y la angustia, a una edad en que el desarrollo aún no se ha completado y en que la calidad de la instrucción y educación es significativamente de menor calidad.

La información proveniente del Instituto Médico Legal de Chile revela que la mayor proporción de denuncias por abusos sexuales corresponden a menores de edad y cuanto menor es la edad de las denunciantes, mayor es la ocurrencia de estos abusos en el ámbito intrafamiliar o por conocidos de la familia. Todo esto ocurre en la población de menor nivel socio-económico, de hecho no existe o es una excepción, en las comunas más pudientes 
de la Región Metropolitana o en las familias acomodadas de las Regiones, donde se concentran los mejores niveles de educación de los padres y la mejor calidad de la educación, que permite que haya muy pocos nacimientos en menores de edad o simplemente no existen.

Ya no se puede tapar el sol con un dedo. No ver esta realidad es una desidia e irresponsabilidad que colinda con la inmoralidad. Todos lo sabemos y en especial los que pertenecemos a la elite que ha alcanzado educación y cultura, y que la transmitimos a nuestros hijos y nietos. Todos sabemos y no es un secreto, ni tampoco necesita ya más evidencias, que esto tiene comprobadas soluciones basadas en la educación y los servicios. Lo mismo que hace 50 años decía el Profesor Hugo Behm respecto de la mortalidad infantil, lo podemos colocar en el contexto de nuestra actual realidad, en la fecundidad de las adolescentes.

No es aceptable seguir creyendo que el embarazo en niñas-madres es el producto de una maternidad deseada. Es necesario ser pobre para que esa percepción de maternidad deseada sea hecha carne y realidad. Esa percepción no ocurre cuando se pertenece a una elite de mayor nivel socio-económico o cultural. La hipótesis es falsa y engañosa y más bien sirve para tranquilizar conciencias.

La sexualidad adolescente ha cambiado drásticamente. Los modelos de comportamiento son más abiertos y liberales. Los medios y sistemas de comunicación modernos cruzan a toda la sociedad y no hay métodos para su control. Es absolutamente indispensable que la información y educación sea amplia, abierta e integral.

La calidad de la educación es fundamental para aumentar la calidad de los proyectos de vida y de la autoestima, dos valores fundamentales para el desarrollo juvenil. No basta con la estrategia del garrote y la zanahoria como estímulos para mejorar la educación. La existencia de la educación sexual integrada a la educación curricular es fundamental, y entregada por profesores capacitados, tanto a alumnos, padres y apoderados. La educación sexual ayuda a la familia y a su desarrollo. Ya no se identifica a la educación sexual como estimuladora de la sexualidad irrestricta, no existen evidencias científicas que lo demuestren. Lo que si tiene evidencias, es en el retraso del inicio de las relaciones sexuales coitales, y que previene el ejercicio del sexo de riesgo e inseguro, cuando se decide asumirlo. Esto es una obligación del Estado, pues es atingente a la población socialmente más vulnerable y dependiente.

Es claro que la necesidad de Servicios de Salud exclusivos para adolescentes de ambos sexos es una demanda espontánea cuando se tiene educación sexual. Es comprobable que en Chile y específicamente en Santiago, todo el sector privado de la atención médica tiene servicios especializados para atender adolescentes. Ya han resuelto esta demanda y los inversionistas de las Isapres, se han dado cuenta que el no tenerlos, aumenta los potenciales costos de sus cotizantes al tener embarazos inesperados, con las secuelas antes enumeradas. Sólo basta calcular la inversión que significa tener un recién nacido con muy bajo peso al nacer $(<1.500$ gramos) en una Unidad de Tratamiento Intensivo Neonatal por dos o más meses de internamiento.

Casi ya no existen Clínicas o Centros de Salud privados que no tengan especialistas en Ginecología Infantil y de la Adolescencia, en un 95\% formados en Chile en el concepto de Salud Sexual y Reproductiva en la Adolescencia y casi todos con aprobación de exámenes internacionales en la especialidad. Masa crítica de la más alta calidad. La entrega de servicios incluye todos los aspectos de la atención, y por cierto la entrega y control de la anticoncepción disponible en el país desde 1964 y refrendada por ley a partir de enero de 2010. Sin embargo, en el Sector Público y en especial en la Atención Primaria Municipalizada, no es una prioridad de inversión, en la magnitud y extensión que se necesita. Esperamos que las decisiones del Gobierno en estas materias sean tomadas con prontitud y sin sesgos. Todos los especialistas estaremos prontos a colaborar con nuestro país y en especial con la población más vulnerable.

El 4 y 5 de noviembre en Santiago, la Federación Internacional de Obstetricia y Ginecología (FIGO) ha organizado una reunión regional con expertos de 7 países de Latinoamérica. El objetivo es revisar el texto de la propuesta de FIGO en servicios para adolescentes en salud sexual y reproductiva. Esta oportunidad permitirá a las actuales autoridades del Ministerio de Salud y de la Sociedades Chilenas de la especialidad, tanto en adultas como en niñas y adolescentes, contrastar nuestra realidad del desarrollo en esta área, con lo ya alcanzado por nuestros hermanos de la Región.

Profesor Dr. Ramiro Molina Cartes. Maestro de la Obstetricia y Ginecología Chilena. 